\title{
CSF gusher in a radiologically normal middle and inner ear anatomy
}

\author{
Aarthi Gopal ${ }^{1}$, Manu Malhotra ${ }^{2 *}$, Saurabh Varshney ${ }^{3}$, Madhu Priya ${ }^{4}$, Abhishek Bharadwaj ${ }^{5}$, Joyson Xavier $^{6}$ \\ ${ }^{1,6}$ Junior Resident, ${ }^{2}$ Additional Professor, ${ }^{3}$ Professor, ${ }^{4}$ Associate Professor, ${ }^{5}$ Assistant Professor, Dept. of Otorhinolaryngology, All India \\ Institute of Medical Sciences, Rishikesh, Uttarakhand, India
}

*Corresponding Author: Manu Malhotra

Email: manumalhotrallrm@gmail.com

\begin{abstract}
Introduction: Cochlear implantation is the most acceptable procedure in rehabilitating a child with profound hearing disability who has gained no benefit from hearing aids. Cerebro-spinal fluid (CSF) gusher from the cochleostomy site is the most common complication faced intra-operatively, however it has been rarely reported in cases with normal radiological anatomy.

Case Report: We present a 4 year old boy taken up for right cochlear implantation for congenital bilateral profound sensorineural hearing loss. A severe CSF gusher was noticed which was identified and successfully managed.

Conclusion: CSF gusher can be seen in cases with normal radiology and hence surgeons must anticipate the same. Meticulous handing and a systematic approach can help us control the leak and also have good surgical outcomes.
\end{abstract}

Keywords: CSF gusher, CSF leak, Cochlear implantation.

\section{Introduction}

Cochlear implantation is considered as one of the accepted and standard treatment in rehabilitating a child with profound hearing loss who has gained minimal or no benefit with heading aids. ${ }^{1}$ Cochlear implantation surgeries require meticulous and systematic study of the high resolution computed tomography (HRCT) of the temporal bone and magnetic resonance tomography (MRI) to detect any congenital outer, middle and inner ear abnormalities, as $20 \%$ of the congenital sensorineural hearing loss (SNHL) have inner ear malformations. ${ }^{2}$

Cerebrospinal fluid (CSF) leak during cochlear implantation ranges from 1 to $5 \% .^{3}$ This not only creates a difficult field for insertion of the electrode array due to refraction caused by fluid but also increases post-operative chances of meningitis. ${ }^{4}$ CSF leak during cochlear implantation is most commonly seen in children with inner ear malformation, most commonly with Mondini's deformity which can be detected in the preoperative HRCT. ${ }^{5}$ CSF leak can be of two types, "gusher" which is high flow CSF leak that rapidly fills the cavity and "oozing" which is an intermittent leaking of CSF. ${ }^{3}$

Literature search with Google scholar on 30th March, 2020, using the keywords 'CSF gusher, CSF leak, normal radiology, cochlear implantation and India was done. It was found that CSF gusher with normal radiology has been reported in India by Vashist S et al. in the year 2016, besides this we could not spot other reports using the above criteria till the date of submission. Here we report a case of CSF gusher during cochlear implantation with a normal pre- operative CT and our surgical management experience with the same.

\section{Case Report}

A 4-year-old boy had been followed up in our out-patient department for complaints of delayed speech which was noticed by his mother since the age of 5 months. The child was born full term with no significant prenatal, natal and postnatal illness. He had normal developmental milestones and was fully immunized as per national guidelines till date. The child then underwent audiological, speech, language and IQ assessment and was found to have bilateral SNHL with normal IQ. The child had no benefit with bilateral hearing aid which he had been using since the age of one year. Radiological assessment including HRCT of the temporal bone and MRI of brain were done and found normal (Fig. 1). No other congenital abnormalities were found on examination. Cochlear implantation of the right ear was planned under general anesthesia.

Post-auricular ' $\mathrm{S}$ ' shaped incision was given and a cortical mastoidectomy was done. After a posterior tympanotomy, the round window niche was exposed through the facial recess approach. Round window was exposed by drilling out the niche and widened with $1 \mathrm{~mm}$ drill. A CSF gusher was noticed. The CSF leak filled up the entire mastoid cavity even after repeated suctioning. To decrease the CSF pressure, the patient was positioned in reverse Trendelenberg position. The patient was also hyperventilated by the anesthetists, end tidal $\mathrm{CO}_{2}$ was decreased to 28 and the leakage site was temporarily covered with large pieces of gel foam. After a span of 20 to 
30 minutes, the cavity was reassessed. All attempts however, could not stop the flow of the CSF leak due to which the insertion of the electrodes was difficult because of the refraction and deflection of the electrodes due to electrostatic force. (Fig. 2 a \& b)

With continuous suctioning, the electrodes were inserted after widening the round window niche. The Nucleus 22 array of electrodes were inserted and the site was first packed with temporalis muscle graft and then sealed with fibrin glue. The last 3 electrodes were left outside due to difficulty in insertion. The post aural wound was closed after fixing the inner device to the well drilled in the cortex with sutures. (Fig. 3 a \& b) Neural response telemetry (NRT) was obtained in 16 electrodes. This could be because of the packing of muscle graft around the electrodes which could have led to the decreased response in last electrodes.

Post- operative x-ray mastoid (Modified Stenver's view) and position of the electrodes were confirmed. (Figure 4) Patient was given intravenous antibiotics and supportive treatment. There was no post-operative complication and was the patient was discharged under satisfactory conditions. During follow-up, patient did not have any vestibular complaints or evidence of CSF leak.

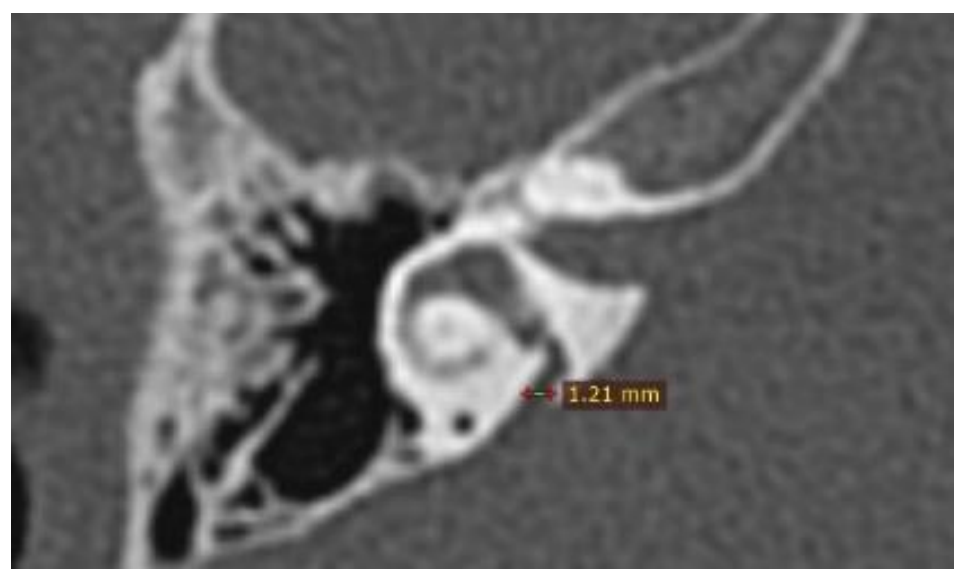

Fig. 1: HRCT of the temporal bone showing normal vestibular aqueduct

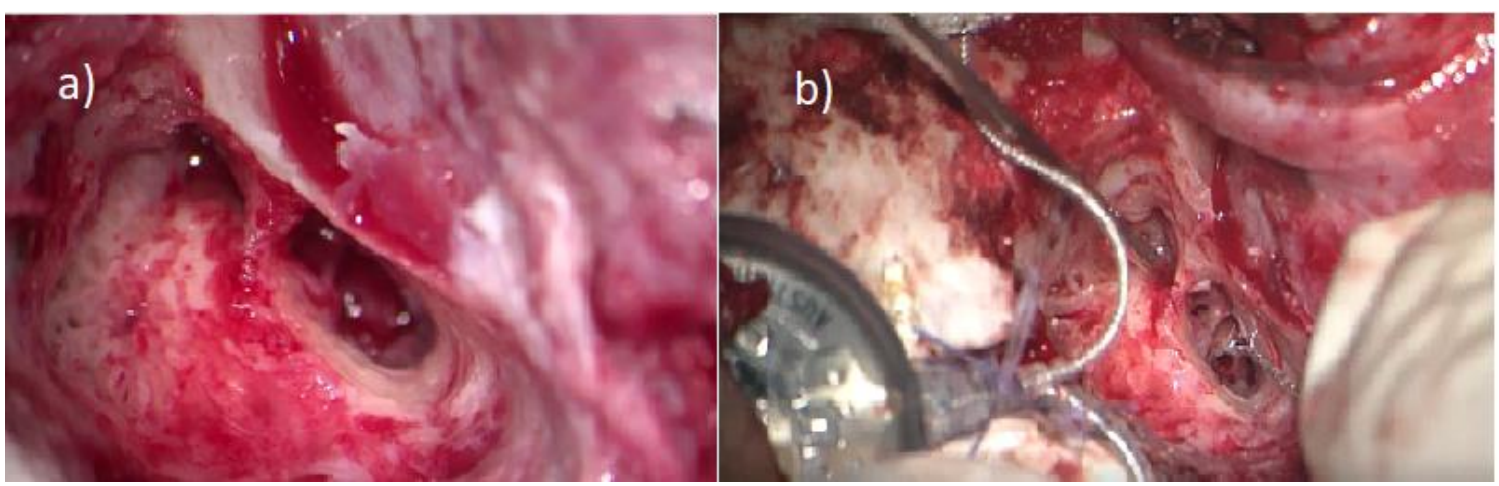

Fig. 2 (a): CSF gusher filling up the mastoid cavity; (b): Insertion of the electrodes through the cochleostomy site

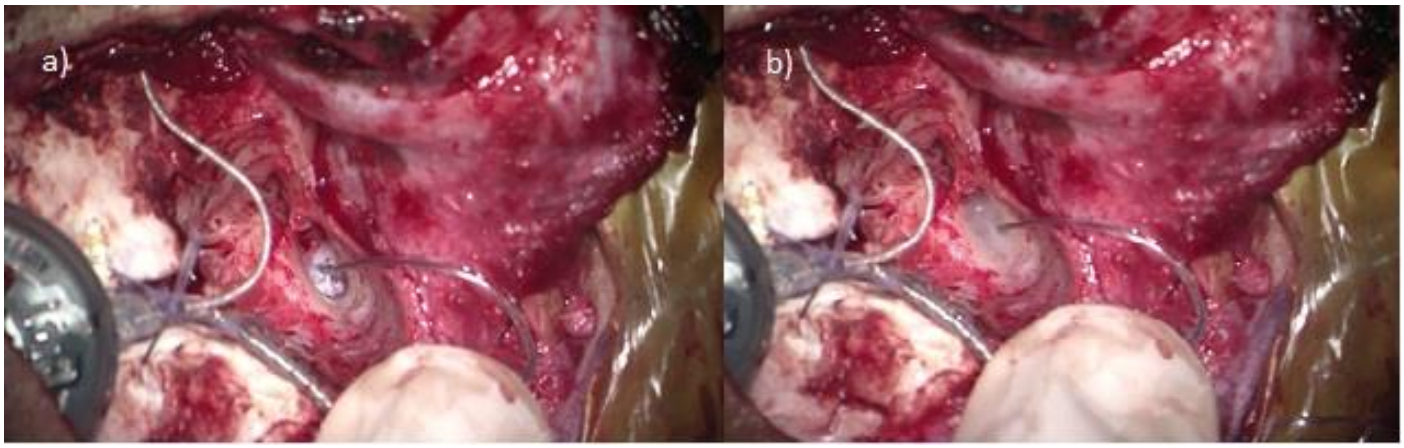

Fig. 3 (a): Packing the cochleostomy site with muscle graft; (b): Application of fibrin glue over the graft 


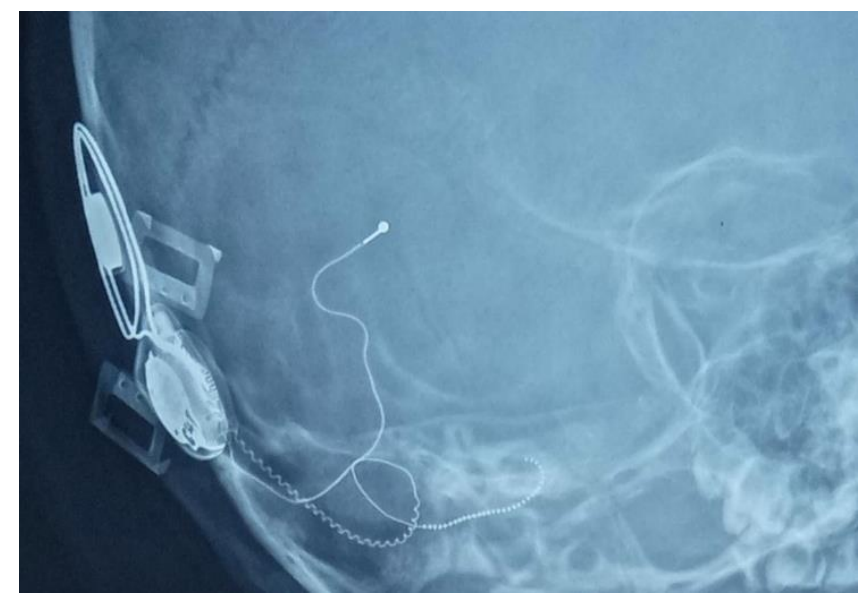

Fig. 4: Post operative X-ray showing straight array of electrodes in the cochlea

\section{Discussion}

CSF in subarachnoid space extends into internal auditory canal till fundus and cribriform plate separates perilymph from CSF. Cochlea does not contain CSF normally, but it may be encountered during surgeries such as cochlear implantation either through a deficient cribriform plate or after traumatic disruption of temporal bone. ${ }^{6}$ Recent publications have identified defect in basal part of cochlea and has also been attributed to membranous anomalies especially in cases with normal pre-operative CT.,4

Several cases with large vestibular aqueduct with CSF gusher have been reported. The vestibular aqueduct is a tiny, bony canal in the otic capsule that originates on the medial wall of the vestibule and extends toward the cerebellar face of the petrous pyramid at the posterior cranial fossa. It contains the endolymphatic duct connecting the endolymphatic sac and the vestibule. It has an average diameter of 0.6 to $1.5 \mathrm{~mm}$ at its midpoint between the common crus and its opening at the posterior cranial fossa. ${ }^{7}$ The first case of CSF leak was reported by Miyamoto et al. in $1986 .{ }^{3}$ CSF gusher in cases with normal pre-operative CT ranges from 1.5-3\%. ${ }^{7}$ CSF gusher has been graded into simple, mild, moderate and severe depending upon their severity by Mehanna (2015). First or 'simple' category included cases where the insertion of the electrode array stops the CSF gusher while second or mild category included cases which required sealing of the cochleostomy site with periosteum, third (moderate) category included cases which required packing of the basal turn around the electrode array and severe gusher which included cases which required packing of the whole middle ear and closure of the eustachian tube. ${ }^{8}$

Management of a CSF gusher includes giving a head end elevation which is also known as the reverse Trendelenburg position, hyperventilating the patient which causes decrease in cardiac output thereby reducing the CSF pressure, starting on intravenous mannitol to reduce the CSF pressure and decreasing the end tidal $\mathrm{CO}_{2}$ below 28 which leads to cerebral vasoconstriction. ${ }^{6}$ Intraoperative management includes insertion of the electrode array through the cochleostomy site. The cochleostomy site can be plugged with temporalis muscle, fascia, fat or periosteum. ${ }^{3,6,7}$ Periosteum or fascia is preferred over muscle or fat because of the reduced chances of atrophy. Cochleostomy site is finally sealed with fibrin glue. ${ }^{3}$ Bone wax silk suture material has also been used. ${ }^{9}$ A lumbar drain might be required to the post-operative period along with oral acetazolamide. ${ }^{3,6}$ The last resort is a subtotal petrosectomy which allows isolating the middle ear cleft from the inner ear. ${ }^{3}$ There has been a debate on the size of the cochleostomy created. Many authors suggest that the size of the cochleostomy should be small to reduce the CSF leak while the rest suggest having a wide cochleostomy to allow instrumentation. ${ }^{9}$ We recommend a wider cochleostomy as it allows better instrumentation, prevents kinking of the electrodes and damaging the spiral lamina and modiolus.

\section{Conclusion}

CSF gusher in cases of normal CT is rare but can be encountered during cochlear implantation surgeries. Hence, each case has to be approached with caution. Systematic study of the pre-operative HRCT helps us rule out and inner ear malformations and in case of a CSF leak the abovementioned techniques could be tried after assessing the severity of the CSF leak.

\section{Source of Funding}

None.

\section{Conflict of Interest}

None.

\section{References}

1. Raghunandhan S, Kameswaran M, Anand Kumar RS, Agarwal AK, Hossain MD. A study of complications and morbidity profile in cochlear implantation: The MERF Experience. Indian J Otolaryngol Head Neck Surg. 2014;66:161-8.

2. Grover M, Sharma S, Bhargava S, Singh SN, Gupta G, Sharma MP. Cochlear implantation in children with anomalous cochleovestibular anatomy: Our experience. Indian J Otolaryngol Head Neck Surg. 2017;69:504-8.

3. Vashist S, Singh S. CSF Gusher in Cochlear Implant Surgerydoes it affect surgical outcomes? Eur Ann Otorhinolaryngol Head Neck Dis. 2016;133:S21-4.

4. Cabbarzade C, Sennaroglu L, Süslü N. CSF gusher in cochlear implantation: The risk of missing CT evidence of a cochlear 
base defect in the presence of otherwise normal cochlear anatomy. Cochlear Implants Int. 2015;16:233-6.

5. Hongjian L, Guangke W, Song M, Xiaoli D, Daoxing Z. The prediction of CSF gusher in cochlear implants with inner ear abnormality. Acta Otolaryngol. 2012;132:1271-4.

6. Chauhan VM, Vishwakarma R. CSF gusher and its management in cochlear implant patient with enlarged vestibular aqueduct. Indian J Otolaryngol Head Neck Surg. 2019;71:315-9.

7. Pradhananga R, Natarajan K, Devarasetty A, Kameswaran M. Cochlear implantation in isolated large vestibular aqueduct syndrome: Report of Three Cases and Literature Review. Int Arch Otorhinolaryngol. 2015;19:359-63.
8. Mehanna AM, Fathala MF, Elwany MS. A stepwise algorithm for the management of cerebrospinal fluid gusher during cochlear implantatio. Egypt J Otolaryngol. 2015;31:19-29

9. Eftekharian A, Amizadeh M. Cerebrospinal fluid gusher in cochlear implantation. Cochlear Implants Int. 2014;15:179-84.

How to cite this article: Gopal A, Malhotra M, Varshney S, Priya M, Bharadwaj A, Xavier J. CSF gusher in a radiologically normal middle and inner ear anatomy. IP Indian J Anat Surg Head Neck Brain 2020;6(1):32-5. 